\title{
Résolution numérique en volumes finis d'un système d'équations de Serre étendu
}

\section{Rodrigo Cienfuegos* ${ }^{*}$ Eric Barthélemy* - Philippe Bonneton**}

* Laboratoire des Écoulements Géophysiques et Industriels BP53 F-38041 Grenoble Cedex 9, France racienfu@ing.puc.cl - eric.barthelemy@hmg.inpg.fr

** Département de Géologie et d'Océanographie

Université Bordeaux I

Av. des Facultés F-33405 Talence, France

p.bonneton@epoc.u-bordeaux1.fr

RÉSUMÉ. Un schéma de résolution numérique en volumes finis pour un système d'équations de Serre étendu est présenté. En utilisant des solutions analytiques tests nous montrons que celuici fournit une erreur de discrétisation globale de $\left\{O\left[(\Delta x)^{4}\right], O\left[(\Delta t)^{4}\right]\right\}$. Le traitement numérique des conditions aux limites de trait de côte mobile et d'absorption-génération d'ondes au large doit permettre d'aborder l'étude de la propagation de la houle sur une plage. La comparaison des résultats numériques avec des mesures expérimentales de propagation d'ondes solitaires sur une plage confirme la capacité des équations de Serre à reproduire les non-linéarités associées au raidissement des ondes dans la zone de levée et l'amplitude limite de celles-ci.

ABSTRACT. A numerical model solving an extended system of Serre equations using the finite volume method is presented. Comparisons with some analytical solutions show that the numerical model reaches a global discretization error of $\left\{O\left[(\Delta x)^{4}\right], O\left[(\Delta t)^{4}\right]\right\}$. The numerical implementation of moving shoreline and absorbing-generating boundary conditions should be sufficient to move on into realistic wave propagation problems on a beach. Furthermore, comparisons between numerical and experimental solitary wave shoaling on a beach confirm the capacity of Serre equations to deal with strong nonlinearities.

MOTS-CLÉS : ondes de gravité, équations de Serre, équations de Boussinesq, modèles numériques, schéma des volumes finis.

KEYWORDS: gravity waves, Serre equations, Boussinesq equations, numerical models, finite volume method. 


\section{Introduction}

Au cours des dernières décennies, d'importants efforts de recherche ont abouti à des améliorations significatives des équations de la famille de Boussinesq qui permettent de décrire la propagation de la houle sur des faibles profondeurs. Ces améliorations concernent en particulier leur extension au large, grâce à diverses techniques de correction de la dispersion linéaire, ainsi que leur comportement non linéaire (e.g. Madsen et Schäffer, 1998; Meftah et al., 2004). Elles constituent à présent un outil mathématique puissant qui permet d'accéder à un certain nombre d'informations indispensables (asymétries des houles, vitesses, etc.) pour une prédiction correcte de l'évolution morphologique des plages. Dans le contexte des applications côtières il est maintenant possible dans ce type de modèles de représenter la levée non linéaire de la houle, la dispersion en fréquence et la dissipation d'énergie par déferlement qui a lieu dans la zone de « surf » (voir Kirby, 2003).

Dans la pratique, la résolution numérique de ce type d'équations a été principalement abordée par des schémas aux différences finies centrées conçus pour minimiser la dispersion numérique qui peut éventuellement l'emporter sur la vraie dispersion physique du système (e.g. Abbott et al., 1984 ; Wei et Kirby, 1995a). Similairement, quelques exemples de l'application de la méthode des éléments finis à ce type d'équations existent (cf. Antunes do Carmo et al., 1993; Woo et Liu, 2001). Cependant, l'utilisation de la méthode des volumes finis n'a été abordée que récemment (e.g. Bradford et Sanders, 2002).

Nous décrivons dans ce travail une résolution numérique des équations de Serre (1953), avec une correction de la dispersion basée sur l'approche décrite dans Madsen et al. (1991), par un schéma de volumes finis à grilles décalées pour la discrétisation spatiale. L'intégration dans le temps s'effectue à l'aide d'une méthode de RungeKutta d'ordre 4. Dans la première partie nous présentons la forme quasi-conservative des équations que nous avons choisies de résoudre numériquement, pour aborder ensuite les aspects pratiques de la mise en œuvre du schéma de résolution. Finalement, nous évaluons les capacités de la nouvelle méthode en comparant ses prédictions avec quelques solutions analytiques et expériences en laboratoire.

\section{Equations de Serre étendues}

En s'intéressant aux ondes longues à forte courbure, Serre (1953) obtient une théorie plus générale que celle décrite par les équations de Boussinesq. Il en déduit alors, sur un fond graduellement varié, un système d'équations moyennées sur la colonne d'eau qui incorpore les effets convectifs associés à une forte accélération verticale du fluide. Plus tard, Seabra-Santos et al. (1987) généralisent les équations de Serre à un fond quelconque. Ces équations constituent en fait un modèle de Boussinesq sans restriction sur les non-linéarités $\left(\epsilon=a / h_{0}\right)$ où tous les termes en $O\left(\sigma^{2}\right)$ sont retenus (avec $\sigma=k h_{0}, a$ et $1 / k$ étant des grandeurs représentatives de l'amplitude et de la longueur de l'onde, tandis que $h_{0}$ est une mesure caractéristique de la profondeur 


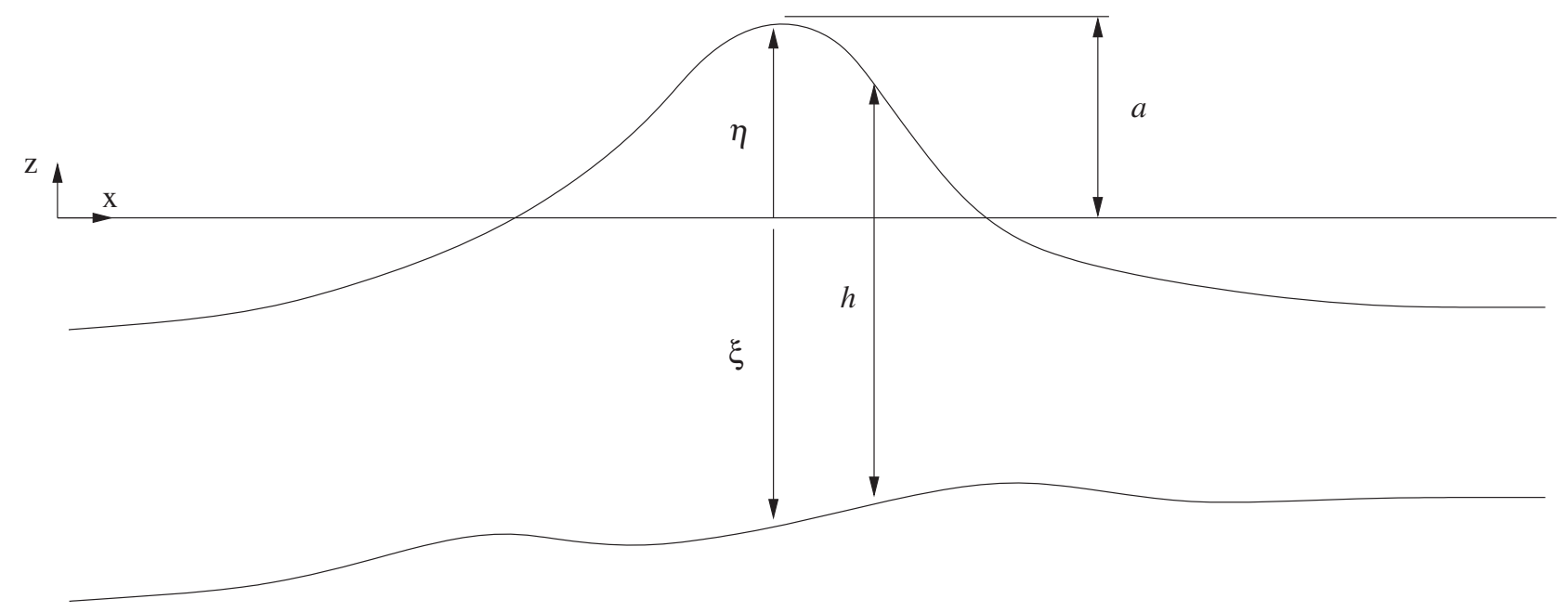

Figure 1. Propagation d'une onde sur un fond quelconque : définition schématique du problème

d'eau). Il s'agit donc d'un système d'équations équivalent (mise à part la stratégie de correction de la dispersion) à celui présenté quelques années plus tard par Wei et al. (1995b).

Dans le cadre des applications côtières, il faut remarquer que les hypothèses qui conduisent aux équations de Serre imposent des restrictions importantes concernant leur validité dans la limite de l'eau profonde. En particulier, la vitesse de phase des ondes courtes $\left(k h_{0} \sim O(1)\right)$ est mal reproduite. En utilisant une approche étudiée par Madsen et al. (1991) qui consiste à rajouter des termes d'ordre $O\left(\sigma^{4}\right)$ afin de modifier la relation de dispersion linéaire du système, il est possible d'étendre la limite d'application des équations de Serre à des eaux plus profondes. Il s'agit de rajouter à l'équation de conservation de la quantité de mouvement le terme,

$$
B(x, t)=-\alpha \xi^{2}\left[u_{x x t}+\left(u u_{x}\right)_{x x}+g(h+\xi)_{x x x}\right],
$$

avec $\alpha$ un coefficient à ajuster. Nous avons introduit ici la coordonnée horizontale $x$ (avec l'axe $O x$ confondu avec la position de la surface libre au repos), le temps $t$, l'équation pour le fond $z=\xi(x)$ (avec $z$ la coordonnée verticale positive vers le haut), la hauteur totale de la colonne d'eau $h(x, t)=\eta(x, t)-\xi(x)$ ( $\eta$ étant la dénivellation de la surface libre), la vitesse horizontale moyennée sur la colonne d'eau $u(x, t)$ et l'accélération de la gravité $g$ (cf. figure 1). 
En incorporant le terme [1] dans les équations présentées par Seabra-Santos et al. (1987), il est possible d'écrire le système d'équations de Serre étendu sous la forme quasi-conservative suivante,

$$
\begin{aligned}
& h_{t}+(u h)_{x}=0 \\
& q_{t}+(u q+F)_{x}=S \\
& q=(1+r) u-\frac{1}{3 h}\left(h^{3} u_{x}\right)_{x}-\alpha \xi^{2} u_{x x}
\end{aligned}
$$

avec,

$$
\begin{aligned}
& F=g(h+\xi)-\frac{1}{2} u^{2}\left(1+\xi_{x}^{2}\right)+u \xi_{x} h u_{x}-\frac{1}{2}\left(h u_{x}\right)^{2}-\alpha \xi^{2}\left[u_{x}^{2}+g(h+\xi)_{x x}\right], \\
& S=-2 \alpha \xi \xi_{x}\left[u u_{x}+g(h+\xi)_{x}\right]_{x} \quad, \quad r=\left(h_{x}+\xi_{x}\right) \xi_{x}+\frac{1}{2} h \xi_{x x} .
\end{aligned}
$$

Nous remarquons que la correction introduite permet d'approximer la relation de dispersion de Stokes à l'aide d'un développement de Padé [2,2] quand $\alpha=1 / 15$ (Barthélemy, 2004).

Il est intéressant de noter que contrairement aux équations de Boussinesq, sur fond horizontal et pour $\alpha=0$, ce système d'équations admet une solution explicite sous forme d'une onde solitaire qui correspond à la solution de Rayleigh. Nous nous servirons plus tard de cette propriété pour étudier l'ordre réel de convergence de notre schéma numérique. En effet, dans le cas général, la résolution du système [2]-[4] doit faire appel au calcul numérique. Or, cette forme conservative des équations de Serre est particulièrement adaptée à une discrétisation en volumes finis et sera exploitée dans la section suivante.

Enfin, il faudra noter que malgré son apparence, il ne s'agit pas ici d'un système d'équations strictement hyperbolique puisque la variable auxiliaire $q$ qui apparaît dans la relation [4] introduit une dépendance horizontale dans l'espace des fonctions caractéristiques, $x-t$. Il s'agit d'une propriété importante puisque $a$ priori ce système ne développera pas de discontinuités (chocs). Cette propriété mathématique traduit le fait qu'un équilibre entre les termes non linéaires et dispersifs peut exister et implique que des solutions d'ondes à forme constante sont admises (Whitham, 1974).

\section{Résolution numérique}

La méthode des volumes finis consiste à diviser l'espace de définition du problème à l'aide de volumes élémentaires et d'intégrer les équations sur chacun d'eux. Les nœuds où les fonctions sont calculées correspondent aux centroïdes de chaque volume de contrôle et les valeurs sur les frontières sont alors interpolées. Les intégrales de surface et de volume doivent être estimées numériquement à l'aide de formules de quadrature. Cette méthode numérique possède des propriétés très intéressantes (Ferziger et Perić, 2002) : i) elle respecte la physique du problème puisqu'elle est conservative par construction, ii) elle peut être facilement adaptée à des problèmes à géométrie 
complexe, et iii) le maillage du domaine nécessite la définition de la position relative des frontières des volumes élémentaires mais n'est pas forcément associé à un système de coordonnées particulier.

Un autre avantage de l'application de la méthode des volumes finis, en particulier au système d'équations [2]-[4], concerne la diminution de l'ordre des dérivées. En effet, l'utilisation d'un schéma standard aux différences finies nécessiterait d'approximer des termes de dérivée de $3^{\mathrm{e}}$ ordre, or avec la résolution en volumes finis l'ordre maximum des dérivées à estimer sera de deux. La principale difficulté associée aux termes d'ordre supérieur est liée à leur discrétisation numérique car les erreurs de troncature du modèle discret peuvent avoir la même forme que certains des termes dispersifs présents dans les équations. Pour pallier cette situation, une discrétisation d'ordre élevée doit être envisagée. Nous choisissons donc de développer un schéma numérique de haute précision avec une erreur de discrétisation $\left\{O\left[(\Delta x)^{4}\right], O\left[(\Delta t)^{4}\right]\right\}$.

\subsection{Discrétisation spatiale : schéma à grilles décalées}

Afin de diminuer le nombre d'interpolations requises et d'améliorer en même temps la résolution numérique des composantes de courte longueur d'onde (cf. Durran, 1999), nous allons intégrer les équations [2] et [3] sur le volume de contrôle $\Omega_{i}$, tandis que l'équation [4] le sera sur le volume $\Omega_{j}$ (cf. figure 2). L'intégration sur les volumes de contrôle respectifs permet d'écrire,

$$
\begin{aligned}
& \frac{d}{d t}\left(\int_{x_{j}}^{x_{j+1}} h d x\right)+\left.(u h)\right|_{x_{j}} ^{x_{j+1}}=0 \\
& \frac{d}{d t}\left(\int_{x_{j}}^{x_{j+1}} q d x\right)+\left.(u q)\right|_{x_{j}} ^{x_{j+1}}+\left.F\right|_{x_{j}} ^{x_{j+1}}=\int_{x_{j}}^{x_{j+1}} S d x, \\
& \int_{x_{i-1}}^{x_{i}} q d x=\int_{x_{i-1}}^{x_{i}}\left[(1+r) u-\frac{1}{3 h}\left(h^{3} u_{x}\right)_{x}-\alpha \xi^{2} u_{x x}\right] d x,
\end{aligned}
$$

avec $\left.f\right|_{x_{a}} ^{x_{b}}=f\left(x_{b}\right)-f\left(x_{a}\right)$. Afin de garantir une erreur de discrétisation $O\left[(\Delta x)^{4}\right]$ nous utilisons la méthode de Simpson pour évaluer les intégrales et une interpolation cubique pour estimer les valeurs des variables sur les frontières de chaque volume de contrôle (cf. Ferziger et Perić, 2002).

L'intégrale des termes non conservatifs de la fonction $S$ dans l'équation [6] et du membre de droite de l'équation [7] doit être approximée. En prenant comme exemple le $2^{\mathrm{e}}$ terme de ce dernier, nous écrivons,

$$
-\int_{x_{i-1}}^{x_{i}} \frac{1}{3 h}\left(h^{3} u_{x}\right)_{x} d x \simeq-\left.\frac{1}{3 h_{j}}\left(h^{3} u_{x}\right)\right|_{x_{i-1}} ^{x_{i}} .
$$




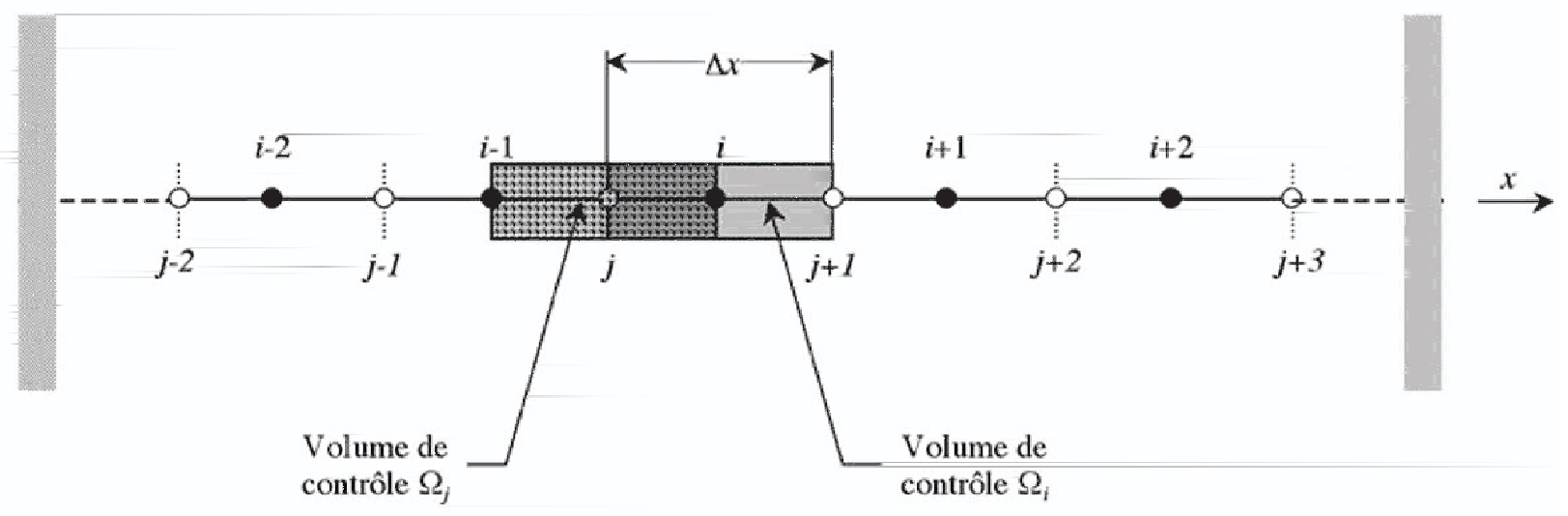

Figure 2. Définition schématique de la grille décalée de discrétisation : h et q sont définis sur les nœuds $i$, u est définie sur les nœuds $j$

Les autres termes sont traités de façon analogue. D'un autre côté, les dérivées par rapport à $x$ sont estimées à l' aide du schéma compact suivant qui fournit une erreur de discrétisation $O\left[(\Delta x)^{4}\right]$,

$$
\left.u_{x}\right|_{i-1}+\left.4 u_{x}\right|_{i}+\left.u_{x}\right|_{i+1}=\frac{3}{\Delta x}\left(u_{i+1}-u_{i-1}\right) .
$$

Enfin, les termes à dérivée seconde sont approximés par application successive du schéma compact.

Le système matriciel global issu du processus de discrétisation spatial est formé de matrices pentadiagonales et peut être exprimé de la manière suivante :

$$
\begin{aligned}
& \mathrm{A} \frac{d \mathbf{h}_{\mathbf{i}}}{d t}+\mathrm{M}\left(\mathbf{u}_{\mathbf{j}}\right) \mathbf{h}_{\mathbf{i}}=0 \\
& \mathrm{~A} \frac{d \mathbf{q}_{\mathbf{i}}}{d t}+\mathrm{M}\left(\mathbf{u}_{\mathbf{j}}\right) \mathbf{q}_{\mathbf{i}}+\mathrm{F}\left(\mathbf{h}_{\mathbf{i}}, \mathbf{u}_{\mathbf{j}}\right)=\mathrm{S}\left(\mathbf{h}_{\mathbf{i}}, \mathbf{u}_{\mathbf{j}}\right), \\
& \mathrm{B} \mathbf{q}_{\mathbf{i}}=\mathrm{G}\left(\mathbf{h}_{\mathbf{i}}\right) \mathbf{u}_{\mathbf{j}} .
\end{aligned}
$$

La discrétisation de l'espace à l'aide de volumes finis nous a donc permis de transformer le système d'équations aux dérivées partielles [2]-[4] en un système d'équations differentielles ordinaires où les matrices $\mathrm{A}$ et $\mathrm{B}$ sont à coefficients constants. Malgré le fait qu'il s'agit de matrices pentadiagonales, l'inversion des matrices constantes pourra être effectuée une seule fois au début des calculs. Pour l'inversion de la matrice à coefficients variables, $\mathrm{G}$, une méthode itérative sera utilisée. Cette stratégie rend donc l'application de la méthode intéressante du point de vue numérique. 


\subsection{Intégration dans le temps et conditions aux limites}

Pour l'intégration dans le temps des équations [10] et [11] nous utilisons une méthode de Runge-Kutta d'ordre 4 qui donne en théorie une erreur $O\left[(\Delta t)^{4}\right]$. À la fin de chaque étape de calcul, le vecteur des vitesses nodales $\mathbf{u}_{\mathbf{j}}$ est calculé à partir de l'équation [12]. Cette dernière équation traduit le caractère parabolique du système. On peut montrer, à l'aide d'un développement en petits paramètres, que dans le cas asymptotique de l'eau peu profonde ( $\sigma \rightarrow 0)$, l'équation [12] n'apporte pas d'informations supplémentaires et le système dégénère alors pour prendre la forme des équations de Saint-Vénant écrites en variables primitives.

D'un autre côté et dans le contexte de la modélisation des évolutions morphodynamiques des plages, nous sommes amenés à définir des conditions aux limites appropriées. En particulier nous devons être capables, au large, de faire entrer une houle mais aussi de permettre aux ondes réfléchies par la plage de sortir du domaine sans provoquer de réflexions parasites. De manière analogue pour l'étude de la formation de barres de réflexion, il est indispensable de bien reproduire la dynamique dans la zone de «swash », en particulier le « runup » et le « run-down».

La mise en œuvre numérique de telles conditions aux limites est un sujet complexe qui ne pourra pas être traité ici. Nous signalons simplement que la condition d'absorption-génération d'ondes au large est basée sur une décomposition quasi-hyperbolique du système de Serre qui permet l'utilisation des équations caractéristiques à condition que pour l'onde sortante, $O\left(\epsilon \sigma^{2}\right) \ll 1$ (cf. Van Dongeren et Svendsen, 1997). Or, dans le cas de réflexion sur une plage à pente relativement douce, cette condition est souvent vérifiée.

Dans la zone de «swash », le traitement du trait de côte mobile s'effectue en utilisant une technique d'extrapolation similaire à celle développée par Lynett et al. (2002). Cette approche s'avère efficace et très facile à mettre en œuvre.

\section{Validation}

\subsection{Propagation d'une onde solitaire sur fond horizontal}

En utilisant la solution analytique d'onde solitaire des équations de Serre, nous étudions ici l'ordre de convergence réel du schéma numérique. Cette solution s'écrit (Seabra-Santos et al., 1987),

$$
\begin{aligned}
& \eta=a \operatorname{sech}^{2}\left[\left(\frac{3 a}{4 h_{0}\left(1+\frac{a}{h_{0}}\right)}\right)^{\frac{1}{2}} \frac{\left(x-c t+x_{0}\right.}{h_{0}}\right], \\
& u=\frac{c \eta}{h_{0}+\eta}
\end{aligned}
$$



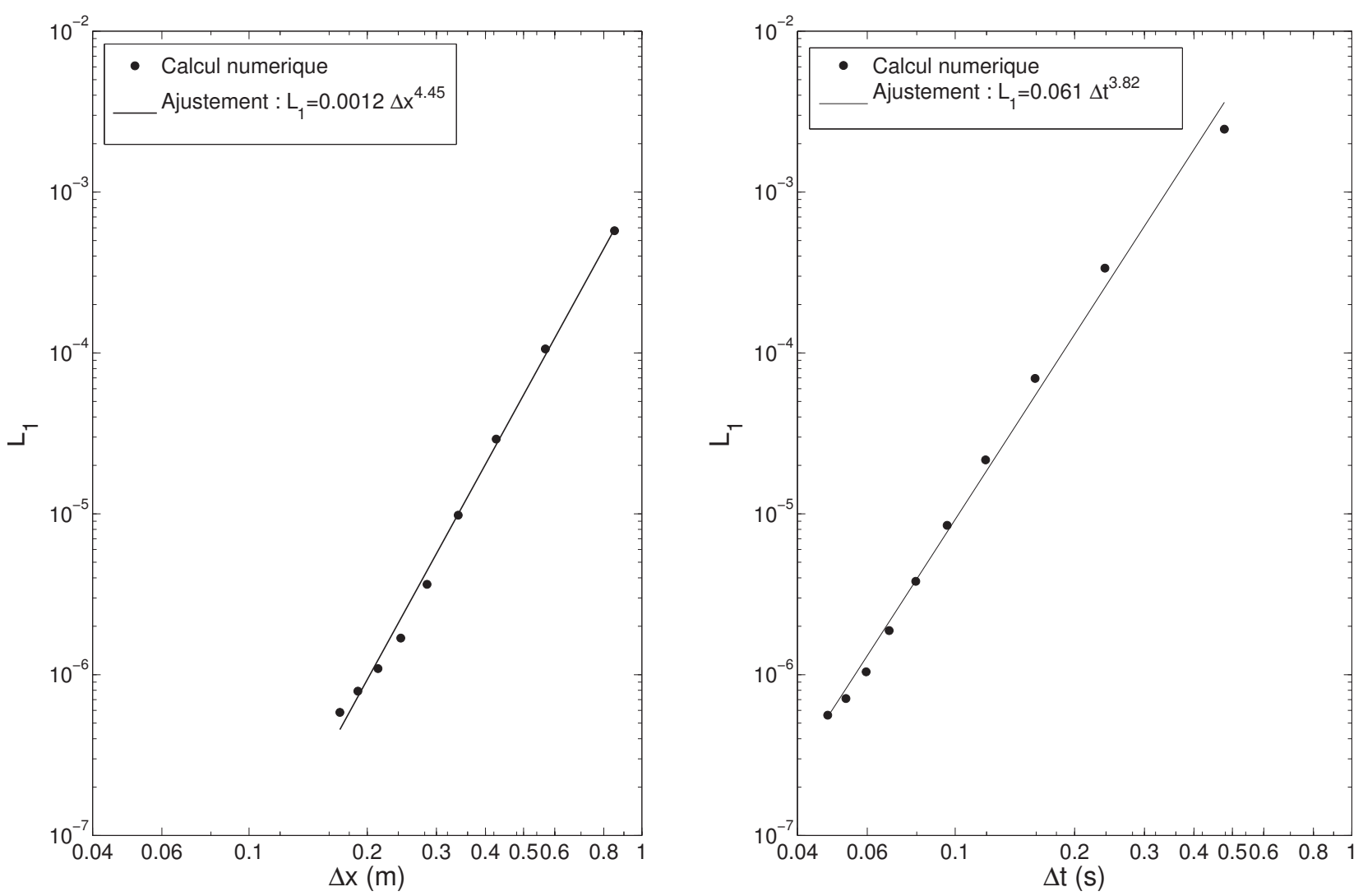

Figure 3. Erreur de discrétisation $L_{1}$ en fonction de $\Delta x$ et $\Delta t$ pour la propagation d'une onde solitaire à a $/ h_{0}=0.3$ sur deux longueurs caractéristiques $\lambda$

avec $a$ l'amplitude de l'onde, $c=\left[g h_{0}\left(1+a / h_{0}\right)\right]^{\frac{1}{2}}$ sa vitesse de propagation, $h_{0}$ la profondeur d'eau au repos, et $x_{0}$ la coordonnée horizontale de la position de l'onde lorsque $t=0$. L'ordre de convergence du schéma numérique peut être alors estimé en étudiant l'erreur commise lorsqu'une onde solitaire d'amplitude relative $a / h_{0}=0.3$ se propage sur deux longueurs caractéristiques $\lambda$ (distance pour laquelle $\eta / h_{0} \geq 0.001$ ), et pour différents pas de discrétisation. L'erreur est estimée à l'aide de la norme,

$$
L_{1}=\frac{1}{N} \sum_{i=1}^{i=N}\left|\eta_{i}^{a}-\eta_{i}^{n}\right|,
$$

avec $\eta^{a}$ la solution analytique et $\eta^{n}$ la solution estimée numériquement. Les résultats sont présentés sur la figure 3 .

Le calcul confirme l'ordre de convergence théorique du schéma en $O\left[(\Delta x)^{4}\right]$ et $O\left[(\Delta t)^{4}\right]$. En effet, lorsque l'on diminue le pas de discrétisation spatial, $\Delta x$, l'erreur associée diminue à la puissance 4.45 , alors que pour une diminution du pas temporel, $\Delta t$, l'erreur suit une courbe de puissance 3.82. La discrétisation spatiale mise en œuvre à l'aide de la méthode des volumes finis s'avère donc très performante en four- 

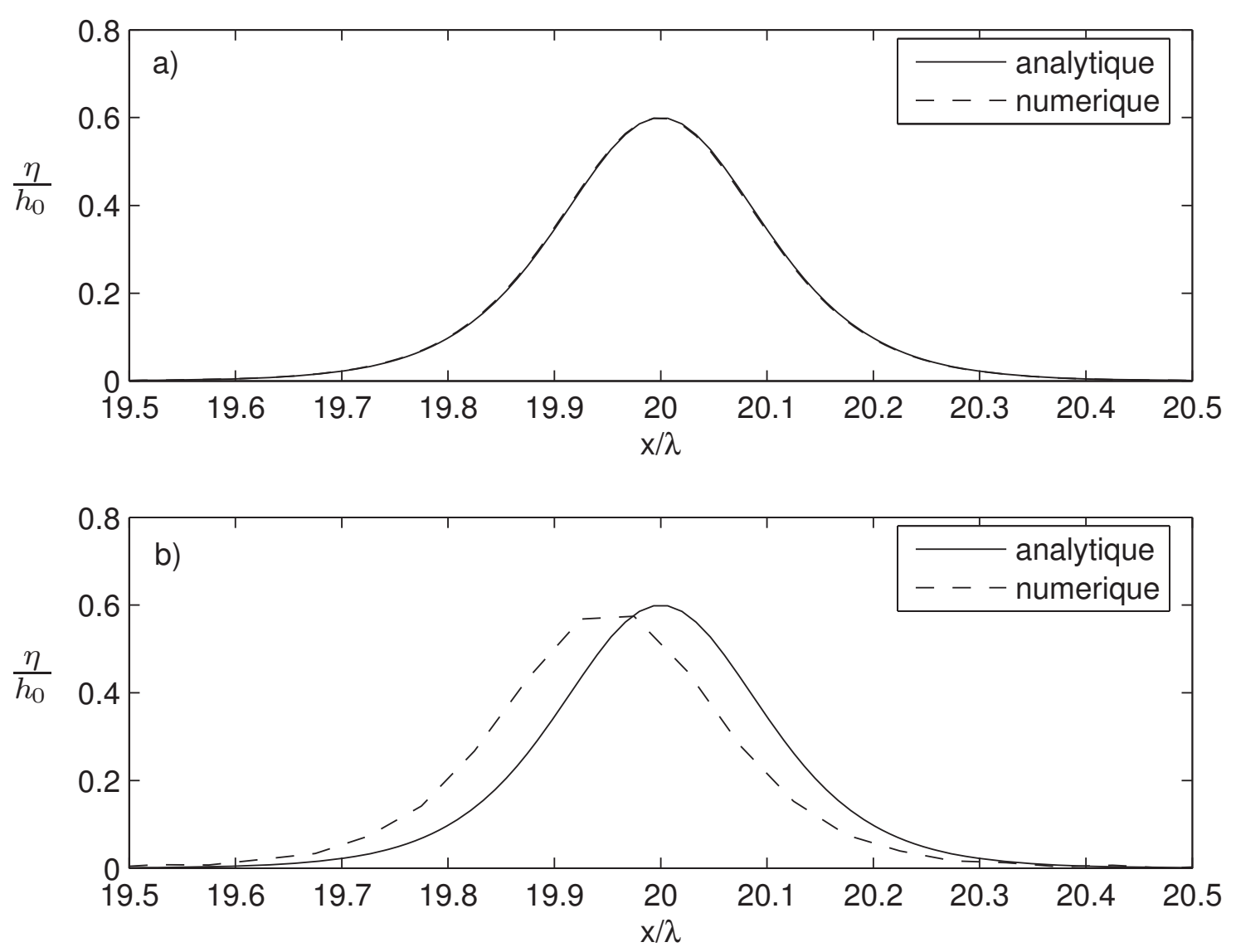

Figure 4. Comparaison de la solution analytique et numérique pour la propagation d'une onde solitaire à a/ $h_{0}=0.6$ sur vingt longueurs caractéristiques $\lambda$. a) Discrétisation avec $\Delta x=\lambda / 75$ et $\Delta t=\lambda /(75 c) ; b)$ discrétisation avec $\Delta x=\lambda / 20$ $\Delta t=\lambda /(75 c)$

nissant une convergence supérieure à la valeur théorique. Cette importante propriété montre que notre schéma est supérieur à d'autres versions publiées précédemment, et en particulier au schéma aux différences finis de Wei et Kirby (1995a) qui utilise une discrétisation au $4^{\mathrm{e}}$ ordre pour les dérivées premières et une discrétisation d'ordre deux pour les dérivées d'ordre supérieur. Or, globalement ce dernier restera d'ordre deux, ou dans le meilleur des cas, il atteindra une valeur comprise entre deux et quatre.

Une autre propriété importante à tester concerne la capacité du schéma à conserver la forme et la célérité d'une onde solitaire au cours du temps. Pour évaluer cette propriété nous propageons une onde solitaire fortement non linéaire $\left(a / h_{0}=0.6\right)$ sur une vingtaine de longueurs caractéristiques, $\lambda$. Les résultats présentés sur la figure 4 montrent qu'avec une résolution spatiale $\Delta x=\lambda / 75$ la forme et la célérité de l'onde sont très bien reproduites par la méthode numérique puisque l'accord entre la solution analytique et le calcul est excellent. En utilisant une discrétisation spatiale plus grossière $(\Delta x=\lambda / 20)$, un déphasage entre la position théorique de l'onde et celle prédite par le calcul apparaît. Cependant, l'erreur relative de cet écart est inférieure à $0.5 \%$ et 


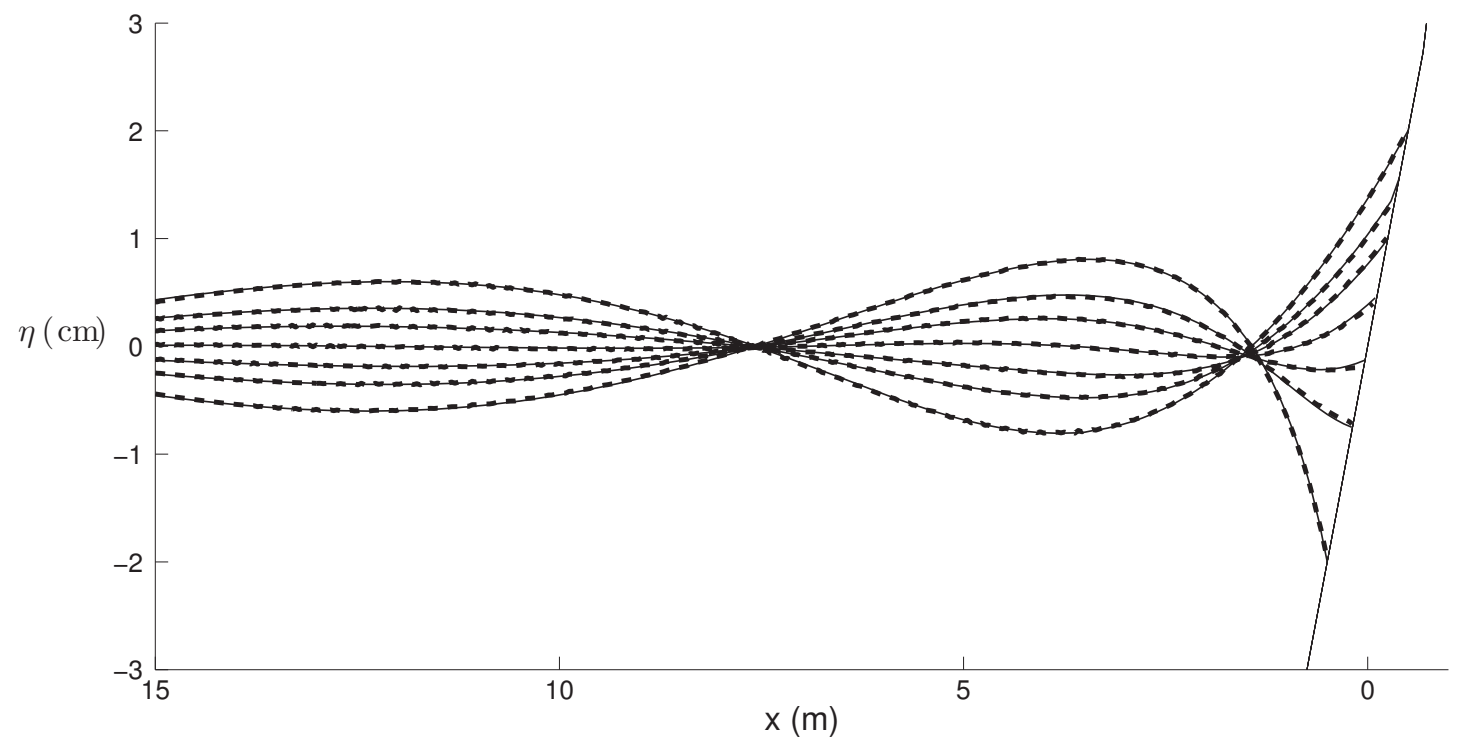

Figure 5. Déplacements de la surface libre de l'onde stationnaire. Théorie de Carrier et Greenspan (1958) (-) et résolution numérique des équations [2]-[4] (- -) avec $\Delta x=0.04$ m et $\Delta t=0.02 \mathrm{~s}$

l'attenuation de l'amplitude de l'onde est très faible elle aussi. Des tests similaires ont été effectués par Wei et Kirby (1995a), avec un schéma aux différences finies, et par Woo et Liu (2001) en utilisant un schéma basé sur la méthode des éléments finis. La performance du schéma aux volumes finis développé ici semble supérieure même si une comparaison directe ne peut pas être effectuée puisque ces auteurs ont testé leur codes avec des ondes solitaires faiblement non linéaires avec des amplitudes relatives comprises entre $a / h_{0}=0.1$ et $a / h_{0}=0.3$. La résolution numérique en volumes finis est donc très peu dissipative et conserve bien la forme et la célérité d'une onde solitaire fortement non linéaire même pour une discrétisation de $\Delta x=\lambda / 20$.

\subsection{Validation du traitement des conditions aux limites}

Ici, la solution analytique de Carrier et Greenspan (1958) qui décrit une onde stationnaire non déferlante sur une plage à pente uniforme sera utilisée pour valider à la fois la condition aux limites de trait de côte mobile et d'absorption-génération d'ondes au large. Pour cela, nous faisons entrer une onde sinusoïdale d'amplitude $a=0.3 \mathrm{~cm}$ et de période $T=10 \mathrm{~s}$ dans une section à fond horizontal d'un canal qui comporte une plage de pente $1 / 25$. La condition limite au large se situe à $30 \mathrm{~m}$ de la ligne de côte où la profondeur d'eau au repos est de $h_{0}=50 \mathrm{~cm}$ (la pente commence à $12.5 \mathrm{~m}$ de la côte). Le calcul se poursuit alors jusqu'à atteindre la solution stationnaire qui est la conséquence de la superposition d'ondes incidentes et réfléchies. Les termes dispersifs ne sont pas pris en compte dans le calcul afin de pouvoir comparer les résultats numériques avec la solution analytique. On observe sur la figure 5 une concordance remarquable entre la théorie et le calcul. Ce résultat montre que les conditions aux 

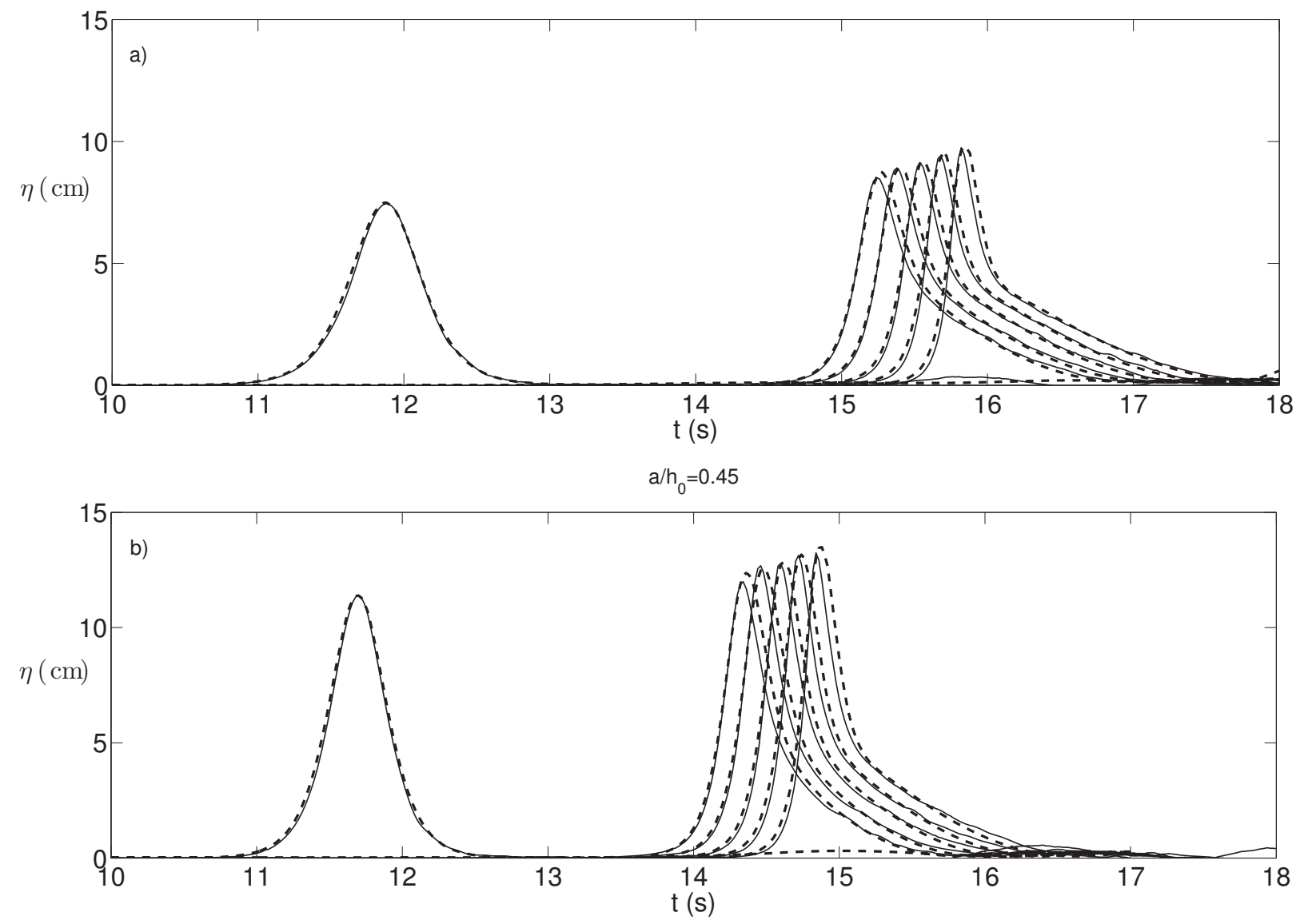

Figure 6. Déplacement de la surface libre mesuré (-) et calculé numériquement $(--)$ dans différentes sections du canal. a/ $h_{0}$ correspond à l'amplitude relative de l'onde incidente (mesurée par la $1^{\text {ère }}$ sonde située à une distance $x=9.94 \mathrm{~m}$ du trait de côte). Localisation des autres sondes : a) $x=3.980,3.765,3.510,3.290,3.052$ $m$; b) $x=4.910,4.695,4.444,4.220,3.982 m$

limites utilisées permettent de bien représenter le « runup » et le « run-down » ainsi que d'absorber les ondes parasites résultantes de la réflexion sur la plage. D'autres tests effectués avec des ondes de plus forte amplitude confirment que la stratégie numérique adoptée pour simuler les conditions aux limites sur un profil de plage permet de bien représenter une large gamme de situations réalistes.

\subsection{Levée non linéaire d'une onde solitaire}

Le dernier test que nous effectuons consiste à propager une onde solitaire sur une plage de pente uniforme $1 / 30$ et de profondeur $h_{0}=25 \mathrm{~cm}$ dans la partie à fond horizontal. Nous confrontons le calcul numérique à quelques expériences réalisées dans le canal à houle du LEGI $(36 \mathrm{~m})$. Les mesures des déplacements de la surface libre s'effectuent à l'aide de six sondes résistives réparties dans le canal (la 1re située sur la partie à fond horizontal et les 5 autres dans la zone de levée). Il s'agit ici d'un test 
important concernant la performance du modèle en présence de fortes non-linéarités. On note dans la figure 6 un excellent accord. Ce résultat confirme donc la capacité des équations de Serre à prédire l'évolution des ondes dans la zone de levée même dans la limite du déferlement où les non-linéarités sont importantes. En effet, des tests supplémentaires montrent que la déformation non linéaire d'ondes solitaires dans la zone de levée est en général correctement reproduite par ce système d'équations (cf. Barthélemy, 2004). Cette propriété est essentielle pour bien représenter l'amplitude limite de la houle sur une plage ainsi que la localisation du point de déferlement.

\section{Conclusions}

Une nouvelle méthode de résolution numérique qui utilise une discrétisation en volumes finis a été développée avec succès sur une version étendue des équations de Serre. Ces équations incorporent une correction de la dispersion linéaire du système qui permet de reproduire la vitesse de phase d'ondes se propageant même dans la limite théorique de l'eau profonde $\left(k h_{0}=3 \sim 4\right)$. D'un autre côté, la comparaison de résultats numériques avec la solution analytique d'onde solitaire des équations de Serre montre que la méthode développée est robuste, très peu dissipative et engendre une erreur de discrétisation globale de $\left\{O\left[(\Delta x)^{4}\right], O\left[(\Delta t)^{4}\right]\right\}$. La forme et la célérité d'ondes solitaires fortement non linéaires se propageant sur fond horizontal sont très bien reproduites et ne nécessitent pas une très grande résolution spatiale. Ces résultats montrent que la discrétisation en volumes finis utilisée ici est supérieure à d'autres méthodes numériques publiés précédemment (e.g. Wei et Kirby, 2005a; Woo et Liu, 2001). De plus, l'implémentation de conditions aux limites d'absorption-génération au large, et de trait de côte mobile dans la zone de «swash » a été validée et devrait permettre d'avancer vers l'étude de cas plus réalistes.

Finalement, une comparaison entre quelques résultats expérimentaux et numériques dans le cas de la propagation d'ondes solitaires sur une plage à pente uniforme confirme la bonne performance des équations de Serre dans un contexte de forte nonlinéarité. Cette propriété devrait permettre d'obtenir une estimation correcte des amplitudes de houle dans la zone de levée et sera fondamentale pour bien localiser le point de déferlement. Cependant, le problème du déferlement et l'incorporation de la dissipation d'énergie associée dans ces équations doit être abordé afin de disposer d'un modèle hydrodynamique applicable depuis le large et jusqu' au trait de côte. Or, même si quelques exemples d'extension des équations de type Boussinesq en zone de « surf » existent (cf. Schäffer et al., 1993; Kennedy et al., 2000), cette question reste ouverte et davantage de recherches, à la fois expérimentales et numériques, devraient permettre de mieux comprendre et paramétrer ce phénomène. 
Le premier auteur remercie le ministère des Affaires Étrangères français, le CROUS Grenoble, le Centre National pour la Recherche Scientifique du Chili (CONICYT), et l'Université Catholique du Chili pour le soutien financier fourni tout le long de son travail de thèse.

\section{Bibliographie}

Abbott M., McCowan A., Warren I., « Accuracy of short-wave numerical model », J. Hyd. Res., vol. $110, \mathrm{n}^{\circ} 10$, p. 1287-1301, 1984.

Antunes do Carmo J., Seabra Santos F., Barthélemy E., « Surface waves propagation in shallow water : A finite element model », Int. J. Numer. Meth. Fluids, vol. 16, p. 447-459, 1993.

Barthélemy E., « Nonlinear shallow water theories for coastal waves », Surveys Geophys., vol. 25, n 3-4, p. 315-337, 2004.

Bradford S., Sanders B., " Finite-volume models for unidirectional, nonlinear, dispersive waves », J. Watrw. Port Coastal Ocean Eng., vol. 128, n 4, p. 173-182, 2002.

Carrier G., Greenspan H., «Water waves of finite amplitude on a sloping beach », J. Fluid Mech., vol. 4, p. 97-109, 1958.

Durran D., Numerical Methods for Wave Equations in Geophysical Fluid Dynamics, 1ère edn, Springer, 1999.

Ferziger J., Perić M., Computational Methods for Fluid Dynamics, 3ème edn, Springer, 2002.

Kennedy A., Chen Q., Kirby J., Dalrymple R., « Boussinesq modelling of wave transformation, breaking and runup. I : 1D », J. Watrw. Port Coastal Ocean Eng., vol. 126, n 1, p. 39-48, 2000 .

Kirby J., «Boussinesq models and applications to nearshore wave propagation, surfzone processes and wave-induced currents », in, V. Lakhan (ed.), Advances in Coastal Modelling, Elsevier, p. 1-41, 2003.

Lynett P., Wu T., Liu P., « Modeling wave runup with depth-integrated equations », Coastal Eng., vol. 46, p. 89-107, 2002.

Madsen P., Murray R., Sorensen O., «A new form of the Boussinesq equations with improved linear dispersion characteristics », Coastal Eng., vol. 15, p. 371-388, 1991.

Madsen P., Schäffer H., « Higher-order Boussinesq-type equations for surface gravity waves : derivation and analysis », Phil. Trans. Roy. Soc. London, vol. A, n 356, p. 3123-3184, 1998.

Meftah K., Sergent P., Gomi P., «Linear analysis of a new type of extended Boussinesq model », Coastal Eng., vol. 51, n 2, p. 185-206, 2004.

Schäffer H., Madsen P., Deigaard R., « A Boussinesq model for waves breaking in shallow water », Coastal Eng., vol. 20, p. 185-202, 1993.

Seabra-Santos F., Renouard D., Temperville A., « Numerical and experimental study of the transformation of a solitary wave over a shelf or isolated obstacle », J. Fluid Mech., vol. 176, p. 117-134, 1987. 
Serre F., « Contribution à l'étude des écoulements permanents et variables dans les canaux », Houille Blanche, vol. 8, p. 374-388, 1953.

Van Dongeren A., Svendsen I., « Absorbing-generating boundary conditions for shallow water models », J. Watrw. Port Coastal Ocean Eng., vol. 123, n 6, p. 303-313, 1997.

Wei G., Kirby J., « Time-dependent numerical code for extended Boussinesq equations », J. Watrw. Port Coastal Ocean Eng., vol. 121, n 5, p. 251-261, 1995a.

Wei G., Kirby J., Grilli S., Subramanya R., « A fully nonlinear Boussinesq model for surface waves. Part 1. Highly nonlinear unsteady waves », J. Fluid Mech., vol. 294, p. 71-92, 1995b.

Whitham G., Linear and Nonlinear Waves, 1ère edn, Wiley Inter-Science, 1974.

Woo S.-B., Liu P.-F., « A Petrov-Galerkin finite element model for one-dimensional fully nonlinear and weakly dispersive wave propagation », Int. J. Numer. Meth. Fluids, vol. 37, p. 541-575, 2001. 